\title{
The role of VEGF in the diagnosis and treatment of malignant pleural effusion in patients with non-small cell lung cancer (Review)
}

\author{
YAO CHEN ${ }^{1}$, NICHOLAS W. MATHY ${ }^{2}$ and HONGDA LU ${ }^{3}$ \\ ${ }^{1}$ Department of Oncology, Jianghan University School of Medicine, Wuhan, Hubei 430056, P.R. China; \\ ${ }^{2}$ Creighton University School of Medicine, Omaha, NE 68178, USA; \\ ${ }^{3}$ Department of Oncology, Wuhan Central Hospital, Wuhan, Hubei 430014, P.R. China
}

Received October 27, 2017; Accepted February 15, 2018

DOI: $10.3892 / \mathrm{mmr} .2018 .8922$

\begin{abstract}
Malignant pleural effusion (MPE) is a severe medical condition, which can result in breathlessness, pain, cachexia and reduced physical activity. It can occur in almost all types of malignant tumors; however, lung cancer is the most common cause of MPE, accounting for $\sim 1 / 3$ of clinical cases. Although there are numerous therapeutic approaches currently available for the treatment of MPE, none are fully effective and the majority can only alleviate the symptoms of the patients. Vascular endothelial growth factor (VEGF) has now been recognized as one of the most important regulatory factors in tumor angiogenesis, which participates in the entire process of tumor growth through its function to stimulate tumor angiogenesis, activate host vascular endothelial cells and promote malignant proliferation. Novel drugs targeting VEGF,
\end{abstract}

Correspondence to: Dr Yao Chen, Department of Oncology, Jianghan University School of Medicine, 18 Jiangda Road, Wuhan, Hubei 430056, P.R. China

E-mail: chenyao3889@163.com

Dr Hongda Lu, Department of Oncology, Wuhan Central Hospital, 26 Shengli Street, Wuhan, Hubei 430014, P.R. China

E-mail:phlonda@163.com

Abbreviations: CEA, carcinoembryonic antigen; HIF-1, hypoxia-inducible factor-1; IFN, interferon; IL, interleukin; MAPK, mitogen-activated protein kinase; MMP, matrix metalloproteinase; MPE, malignant pleural effusion; NSCLC, non-small cell lung cancer; ORR, objective response rate; PFS, progression-free survival; PI3K, phosphoinositide 3-kinase; PLC, phosphoinositide phospholipase C; RR, overall response rate; STAT, signal transducer and activator of transcription; TNF, tumor necrosis factor; TTF-1, thyroid transfection factor-1; TTP, time to progression; VEGF, vascular endothelial growth factor; VEGFR, vascular endothelial growth factor receptor

Key words: vascular endothelial growth factor, malignant pleural effusion, non-small cell lung cancer, diagnosis, pathogenesis, clinical management, review including endostar and bevacizumab, have been developed and approved for the treatment of various tumors. Data from recent clinical studies have demonstrated that drugs targeting VEGF are effective and safe for the clinical management of MPE. Therefore, VEGF-targeting represents a promising novel strategy for the diagnosis and treatment of MPE. The present review summarized recent advances in the role of VEGF in the pathogenesis, diagnosis and clinical management of MPE in patients with non-small cell lung cancer.

\section{Contents}

1. Introduction

2. Diagnostic value of VEGF for MPE in patients with NSCLC

3. The role of VEGF in the pathogenesis of MPE

4. VEGF-targeted strategies for the management of MPE in patients with NSCLC

5. VEGF in the prognosis of MPE in various subtypes of NSCLC and in patients with advanced NSCLC

6. Conclusion and perspectives

\section{Introduction}

In developing countries, the morbidity and mortality of lung cancer has grown rapidly with the increasing prevalence of smoking and worsening air pollution. The most recent epidemiological data indicate that in the majority of unindustrialized regions, lung cancer is the main cause of mortality among the most common malignant tumors (1). According to data in the 'China Cancer Registration Annual Report 2015' released by the Chinese National Cancer Center, lung cancer is the most common malignant disease, which accounts for $\sim 1 / 4$ of all cancer cases in adult men in China (2). Non-small cell lung cancer (NSCLC) is the most common type of lung cancer, representing $~ 85 \%$ of all lung cancer cases (1). Unfortunately, the majority of patients with lung cancer $(\sim 70 \%)$ are diagnosed at a late stage, and NSCLC accounts for $80 \%$ of these cases (2). Malignant pleural 
effusion (MPE) is one of the most common complications of advanced NSCLC, which is diagnosed by the presence of malignant cells in pleural effusion $(3,4)$. The occurrence of MPE in patients with NSCLC often indicates an average life expectancy of $\sim 3.3$ months, depending on the subtypes of the tumor and its clinical stage $(3,4)$. There is strong evidence to suggest that patients with lung cancer and MPE have a shorter survival time, whereas patients with MPE caused by other malignant diseases, including ovarian cancer and carcinoma of unknown primary, usually survive longer $(3,4)$. Current therapeutic approaches for MPE in patients with NSCLC include chest puncture drainage, thoracic catheter drainage and intracavity chemotherapy $(3,4)$; however, the clinical effects of these approaches are often poor and unsatisfactory.

Vascular endothelial growth factor (VEGF) is a family of endothelial growth factors, which includes VEGFA, $-B,-C,-D$ and $-E$, and placental growth factor (5). As early as 1939 , Ide et al (6) speculated that tumor cells may produce and release proangiogenic factors. Recognizing the role of the tumor microenvironment in tumorigenesis, in 1971, Folkman (7) proposed that 'tumor growth is angiogenesis dependent'. In addition, it was suggested that: i) Virtually all tumors would be restricted to a microscopic size in the absence of angiogenesis; ii) tumors would be revealed to secrete diffusible angiogenic molecules; and, iii) tumor dormancy would result from suppressed angiogenesis. In 1983, Senger et al (8) confirmed that the speculated 'vascular permeability factor' is a VEGF. Ferrara and Henzel (9) successfully isolated and purified the first VEGF in 1989 and demonstrated a critical role for VEGF as an important proangiogenic factor. Later in 1992, De Vries et al (10) identified the first receptor for VEGF. VEGF has now been recognized as the most important regulatory factor in tumor angiogenesis, participating in the entire process of tumor growth through its ability to stimulate tumor angiogenesis, activate host vascular endothelial cells and promote malignant proliferation with the increase of local essential oxygen and nutrients for tumor metastasis (11). It has been reported that VEGF is not only the most important angiogenic factor, but also a potent stimulator that increases vascular permeability and triggers endothelial cell migration $(11,12)$. High expression levels of VEGF have been confirmed in various normal human tissues and an increased level of VEGF has been reported in the serum of patients with numerous types of cancer and in pleural effusions due to malignant diseases (12). Specifically, VEGF levels in MPE are closely associated with the clinical prognosis of patients with NSCLC; therefore, VEGF may be a critical pathological factor in the occurrence and development of MPE in patients with NSCLC $(12,13)$. Notably, NSCLC cells can produce and secrete VEGF, promoting pleural effusion formation, angiogenesis and tumor metastatic progression (14). Improved understanding of the pathogenic mechanisms, coupled with novel local and/or systemic administration of drugs targeting VEGF, has the potential to improve the efficacy of current management strategies for MPE. The present review examined the role of VEGF in the pathophysiology, diagnosis and management of MPE in patients with NSCLC.

\section{Diagnostic value of VEGF for MPE in patients with NSCLC}

The gold standard for the diagnosis of MPE remains the detection of malignant cells in pleural effusion or in the tissues of a pleural biopsy $(4,15)$. The MPE diagnostic methods include chest imaging, pleural fluid cytology and detection of tumor markers, pathological evaluation of pleural biopsy and molecular biotechnology (Table I). The sensitivity and specificity of these methods vary and possess certain limitations (4,15-26); more specific diagnostic methods with a higher sensitivity are therefore required.

The diagnostic methods for MPE measure total cell counts, individual cell counts, protein levels, lactate dehydrogenase, glucose and $\mathrm{pH}$, in addition to microbiological and cytological measurements. A recent meta-analysis report summarized these routine tumor markers in pleural effusion for the diagnosis of MPE, including carcinoembryonic antigen (CEA), carbohydrate antigen (CA) 153, CA 19-9, CA 125 and cytokeratin 19 fragment (CYFRA 21-1) (17). In terms of specificity, a higher level of CEA in pleural effusion may rule out the possibility of malignant mesothelioma; CA 153, CA 19-9 and CYFRA 21-1 may have a high specificity but their sensitivity is relatively low (17). However, the combination of two or more tumor markers in pleural effusion can usually increase the sensitivity of the diagnosis (17). Recent developments in modern molecular biological technology have provided novel markers for the diagnosis of MPE. For example, an elevated level of thyroid transfection factor-1 (TTF-1) mRNA was detected in $73.2 \%$ of patients with MPE, with a sensitivity of $93 \%$ and a specificity up to $100 \%$, whereas a high level of TTF-1 mRNA has not been reported in the pleural effusions of non-malignant patients (21-24).

Due to its high level in the pleural effusions of patients with MPE, VEGF has been implicated as an important marker with significant diagnostic value (17). Elevated mRNA expression levels of VEGF and endostatin in pleural effusion are more frequently detected in patients with MPE than in pleural effusions caused by non-malignant diseases (17); the sensitivity and specificity for elevated VEGF mRNA expression in MPE are 82.6 and $84.3 \%$, respectively. The specificity for elevated endostatin mRNA expression in MPE is almost 100\%. A fluorescence in situ hybridization-based approach has recently been established to reliably detect the copy number of VEGF mRNA in pleural cells from patients with MPE (20). In addition, the mRNA expression levels of VEGF in MPE samples from patients with NSCLC are usually significantly increased compared with in pleural effusion samples from patients with non-malignant diseases (17). In addition, increased VEGF mRNA expression, coupled with VEGF receptor (VEGFR) expression in pleural effusion, can significantly increase the diagnostic sensitivity of MPE in patients with NSCLC (18). It has been reported that a combination of elevated levels of VEGF and endostatin in pleural fluid can increase the diagnostic sensitivity of MPE in patients with NSCLC, particularly for the differential diagnosis of tuberculous pleural effusion (19). A combination of elevated levels of VEGF and CEA in pleural effusion can also increase the diagnostic sensitivity of MPE in patients with NSCLC (26). In addition, the serum levels of VEGF are associated with its level in pleural effusions in patients with NSCLC and MPE (17). 


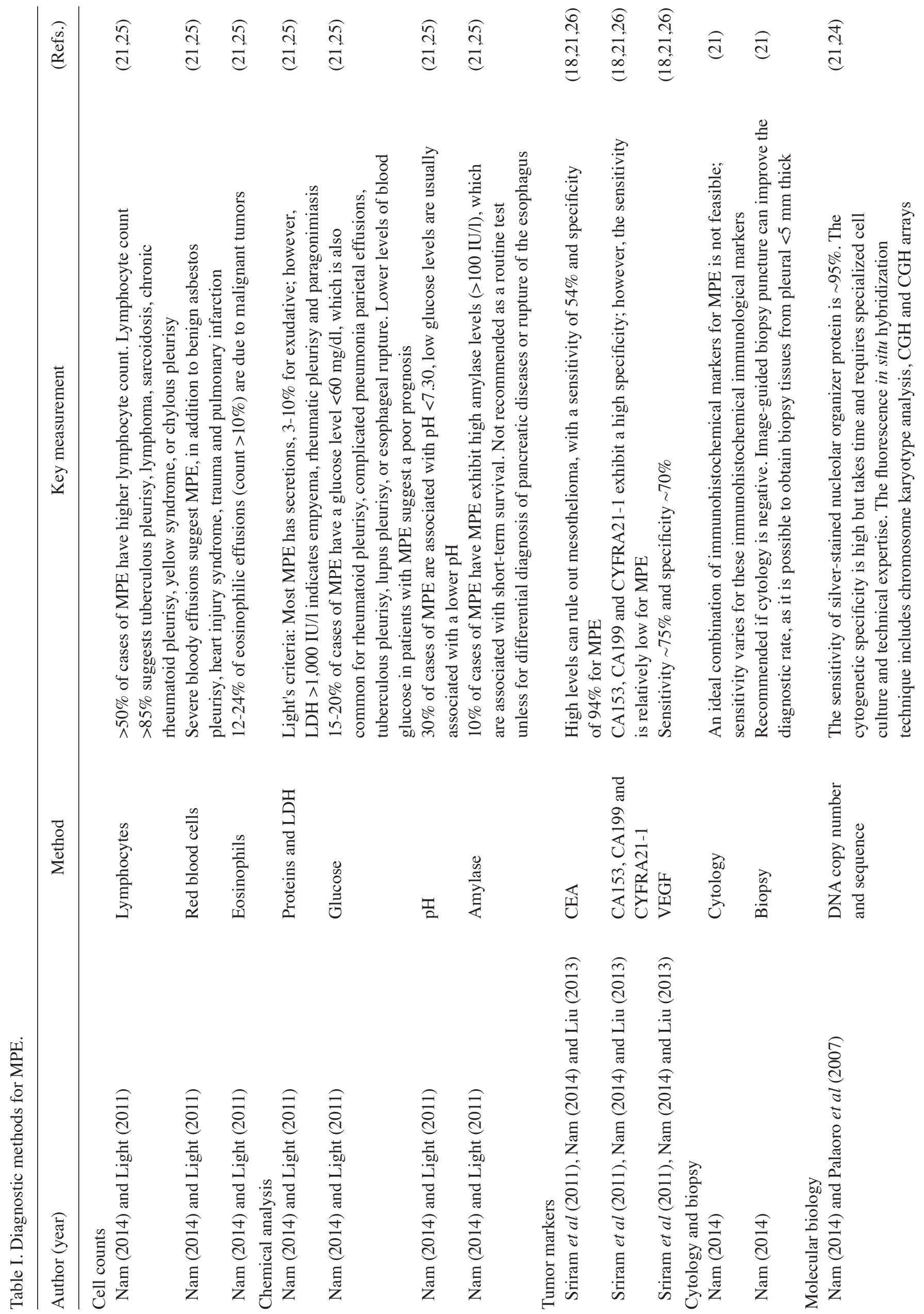




\section{The role of VEGF in the pathogenesis of MPE}

MPE is the result of integrated interactions between host and tumor cells, as summarized by Stathopoulos and Kalomenidis (27). Many effector molecules, from either the host or tumor cells, are involved in its pathogenesis. These effectors can generally be classified into two categories: Immunoregulatory effectors and modulators that increase vascular permeability (Fig. 1). The immunoregulatory factors include interleukin (IL)-2, tumor necrosis factor (TNF) and interferons. Important modulators that induce vascular permeability are VEGF, matrix metalloproteinases (MMPs) and numerous others (28-30). Of these effector modulators, VEGF serves a central role in the accumulation of pleural effusion.

VEGF is a highly conserved homodimeric glycoprotein with a molecular weight ranging between 35 and $44 \mathrm{kDa}$. It has a broad range of biological functions, including stimulation of vascular proliferation, cellular differentiation, migration, survival and germ tube formation, and regulation of vascular permeability and angiogenesis $(5,9,31)$. VEGF has numerous isoforms, including VEGFA, -B, $-\mathrm{C}$ and $-\mathrm{D}$, and placental growth factor in humans, which can specifically bind to one or numerous types of the three VEGFRs (VEGFR1, -2 and -3) $(31,32)$. Upon activation, the VEGFR undergoes autophosphorylation and subsequently activates cell type-dependent signaling cascades, including the phosphoinositide phospholipase $\mathrm{C}$, mitogen-activated protein kinases (MAPKs), nitric oxide synthases and phosphoinositide 3-kinase, in addition to signal transducer and activator of transcription (STAT) 3 and STAT5. Activation of distinct intracellular signaling pathways results in various outcomes associated with the regulation of vascular permeability depending on cell type or state, including induction of inflammatory responses and loss of intracellular integrity and gap formation (31). VEGF-VEGFR interactions can also activate downstream MAPK1 signal cascades to regulate endothelial cell proliferation and migration, and consequently promote tumor angiogenesis and metastatic progression $(9,31)$. Several splicing variants of VEGF have been reported; for example, five VEGF forms of 121-206 amino acids are produced from a single gene by alternative splicing, each with different biological effects to promote neovascularization through distinct mechanisms (33). Therefore, VEGF may promote the occurrence and development of MPE in patients with NSCLC through two integrated mechanisms: By increasing vascular permeability (a direct effect) and by promoting angiogenesis (an indirect effect; Fig. 2).

Elevated VEGF expression has been well demonstrated in various tumor cells in humans, including pancreatic, stomach, colorectal, breast and prostate cancers, melanoma, and cancers of a number of other tissues (34). Patients with various types of lung cancer, particularly those with NSCLC, usually exhibit elevated VEGF expression levels in cancer cells (17-19). Numerous factors in the local tumor environment may contribute to the induction of VEGF expression in tumor cells, including the occurrence of hypoxia and the presence of various growth factors (including epidermal growth factor, transforming growth factor and insulin-like growth factor) and hormones $(35,36)$ (Fig. 2). Among these factors, hypoxia can activate hypoxia-associated transcription factors and 


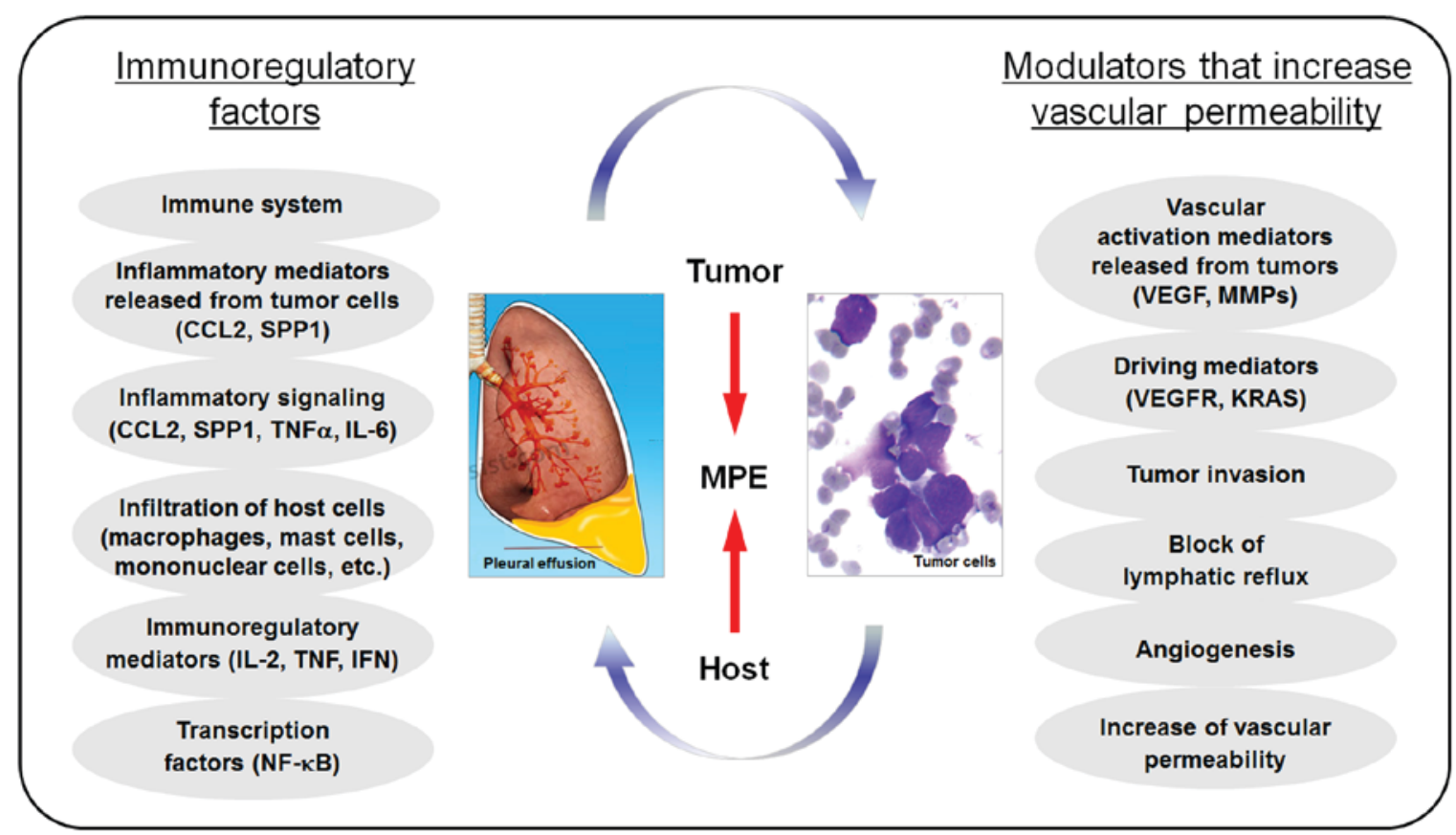

Figure 1. Pathogenesis of MPE. Numerous effector molecules, from either the host cells or tumor cells, are involved in the pathogenesis of MPE. These effectors can generally be classified into two categories. The first group of these effector molecules is important immunoregulatory factors, including IL-2, TNF and IFNs. The second group of effector molecules is effective modulators that increase vascular permeability, including VEGFs, MMPs and numerous others. CCL, C-C motif chemokine ligand; IFNs, interferons; IL, interleukin; KRAS, GTPase KRas; MMPs, matrix metalloproteinases; MPE, malignant pleural effusion; NF, nuclear factor; SPP, S1P phosphatase; TNF, tumor necrosis factor; VEGFs, vascular endothelial growth factors; VEGFR, VEGF receptor.

trigger the transcription of hypoxia-inducible genes (37). One hypoxia-inducible gene is hypoxia-inducible factor-1 $(H I F-1)$; transcription of the $H I F-1$ gene can be induced by hypoxia via transcriptional activation of the mechanistic target of rapamycin (33). Binding of HIF-1 to the regulatory promoter region of the $V E G F$ gene can enhance transcription of the $V E G F$ gene locus (38). Elevated levels of VEGF in the blood and in malignant tissues of patients with solid tumors are often associated with the expression levels of HIF-1 (33). Notably, increased levels of VEGF in MPE may also be caused by interactions between VEGF and VEGFRs, which further stimulate tumor cells and mesothelial cells alike to secrete VEGF (12). IL-6 can trigger transcription of the VEGF gene, whereas $I L-6$ itself appears to be a VEGF-targeted gene in numerous cancer cells $(39,40)$. This autocrine signaling-mediated IL-6 production can further promote the expression of VEGF in cancer, particularly in NSCLC cells (41) (Fig. 2).

\section{VEGF-targeted strategies for the management of MPE in patients with NSCLC}

Current MPE management. Effective management of MPE remains a clinical challenge and current methods for the treatment of MPE in patients with NSCLC include diuretics, limited intake of salt, thoracic puncture, long-term catheter drainage and intrathoracic administration of chemotherapy drugs or biological agents $(3,4)$. There remains a lack of consensus on which approach is most effective and what the dose, interval and course should be for the administration of the drugs $(3,4)$. In addition, identification of the potential long-term effects and the best combination of drugs requires large samples of patients and randomized controlled clinical studies $(3,4)$.
With an improved understanding of the pathogenesis of MPE, particularly the appreciation of a role for angiogenesis in tumor metastatic progression due to the development of vascular-dependent tumor growth theory since 1971, significant advances in the clinical management of MPE in patients with NSCLC have been made in recent years $(3,4,7)$. Novel drugs targeting these effectors and signaling pathways for angiogenesis, coupled with the development of more effective anti-cell proliferation drugs, have provided new strategies for the clinical management of MPE in patients with NSCLC (7). Of these novel drugs, recombinant human endostatin (endostar) and the monoclonal VEGF antibody bevacizumab have demonstrated promising therapeutic benefits for patients with NSCLC and MPE (42).

Endostar to target vascular endothelial cells. In 1997, an endogenous glycoprotein with 184 amino acids (molecular mass, $20 \mathrm{kDa}$ ) was isolated from mouse endosomes and was named endostatin by O'Reilly et al (43). Endostatin has been demonstrated to possess strong antivascular activity, with limited side effects, and was able to almost completely inhibit tumor-induced angiogenesis in murine models (44). Subsequently, a recombinant human endostatin with an additional 9 amino acid sequence (MGGSHHHHH) added to the $\mathrm{N}$-terminal of the protein was engineered; the product of this modification was named endostar (45). Such modification has been reported to significantly enhance the purification, solubility and stability of the protein (45). Endostar also has a longer half-life than endostatin, with much improved medicinal properties and efficacy, resulting in a significant improvement of its biological function (45). It is now a common angiogenesis antagonist used to treat lung cancer; in particular, it is used 


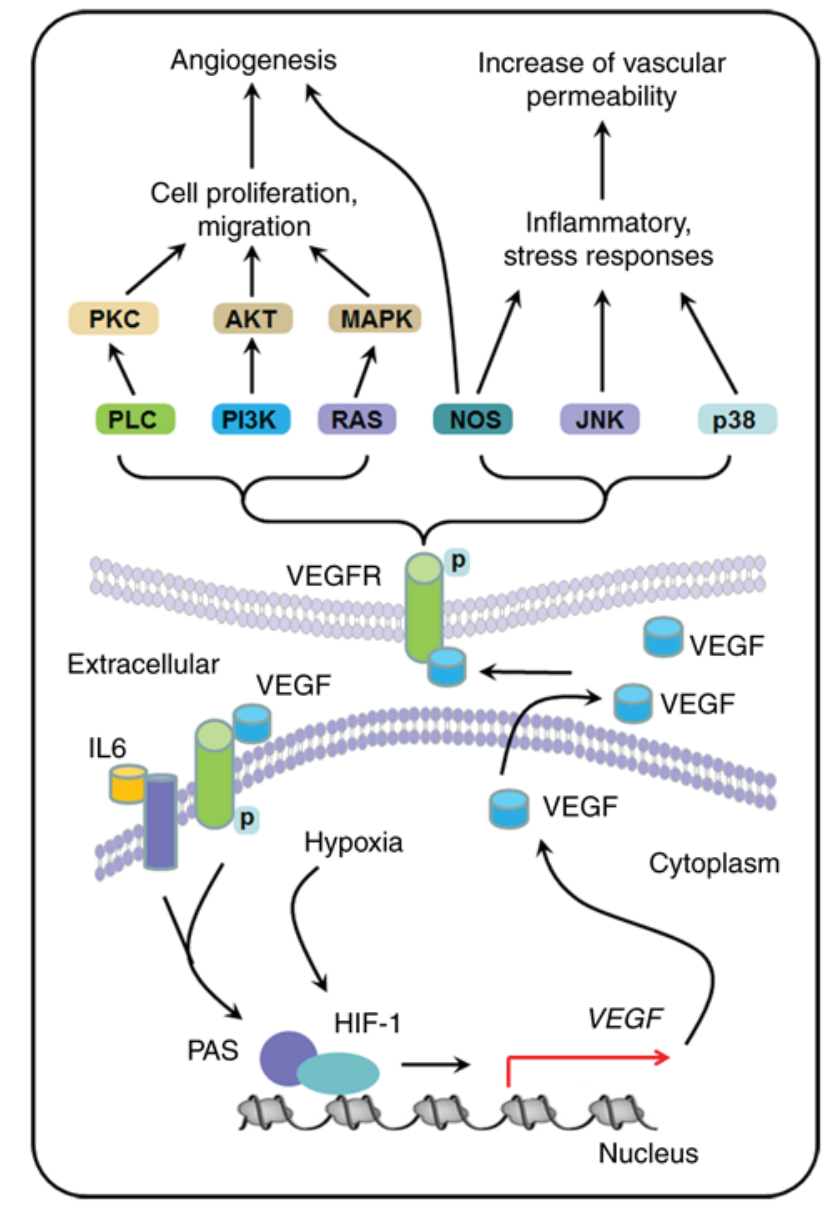

Figure 2. VEGF signaling pathways and their role in the pathogenesis of MPE. VEGF increases vascular permeability and promotes tumor angiogenesis by binding to one of its three receptors VEGFR $1,-2$ and -3 on vascular endothelial cells. Upon activation, the VEGFR undergoes phosphorylation and subsequently activates cell type-dependent signaling cascades, including PLC, PI3K, NOS and MAPKs. Many factors of the local tumor environment may contribute to the induction of VEGF expression in tumor cells, including the occurrence of hypoxia and presence of various growth factors (e.g., VEGF and IL-6). Hypoxia can activate hypoxia-associated transcription factors, including HIF-1, resulting in transcription of the VEGF gene. Through its role in regulation of vascular permeability and angiogenesis, VEGF serves a central role in the accumulation of pleural effusion in tumor patients. HIF-1, hypoxia-inducible factor-1; IL, interleukin; JNK, c-jun NH2-terminal kinase; MAPKs, mitogen-activated protein kinases; MPE, malignant pleural effusion; NOS, nitric oxide synthases; PAS, pathway activation signature; PI3K, phosphoinositide 3-kinase; PKC, protein kinase C; PLC, phosphoinositide phospholipase C; VEGF, vascular endothelial growth factor; VEGFR, VEGF receptor.

for the management of relapse and metastasis in patients with NSCLC (46), and has been widely used in clinical practice to treat other tumors, including squamous cell carcinoma (47).

Antiangiogenic mechanisms of endostatin and endostar. The process of tumor angiogenesis is finely regulated by a complex interaction between proangiogenic growth and antiangiogenic factors $(5,11,12)$. Given the wide variety of antitumor growth effects of endostatin, the following mechanistic themes have been speculated for the antiangiogenic effects of endostatin/endostar. Firstly, endostatin/endostar has been demonstrated to act on the endothelial cells of newly formed blood vessels, inhibit endothelial cell migration and induce endothelial cell apoptosis, thus resulting in limited tumor growth (47). Secondly, endostatin/endostar can downregulate the expression of Neuropilin-1 and VEGFA, which are two potent proangiogenic growth factors in numerous types of tumor due to their actions in triggering endothelial cell proliferation (48). Thirdly, endostatin/endostar can interact with the heparin sulfate proteoglycan receptors and block the receptor binding of proangiogenic growth factors, including VEGF and $\beta$ fibroblast growth factor, thus resulting in inhibition of endothelial cell proliferation and tumor angiogenesis (49). Fourthly, endostatin/endostar has been reported to specifically recognize and bind to integrin $\alpha 5 \beta 1$ and $\alpha \mathrm{V} \beta 3$ on the endothelial cell surface (50). Such interactions may prevent the adhesion of endothelial cells in extracellular matrix-mediated migration, induce tyrosine phosphorylation of adhesion focal kinase and paxillin to promote elastic fiber formation, and consequently inhibit tumor cell adhesion and metastatic progression (50). Finally, endostatin/endostar can inhibit the transcription of $M M P 2$ and $M M P 9$ genes (51). In addition, recent studies using murine models have revealed that overexpression of endostatin may result in downregulation of the $V E G F C$ gene, and inhibition of lymphangiogenesis and tumor metastasis to nearby lymph nodes (52). Therefore, recombinant human endostatin/endostar is a potent antiangiogenic drug with a wide range of potential research prospects.

Endostatin/endostar in the clinical management of MPE in patients with NSCLC. Endostar has been used in clinical practice for numerous years for the treatment of advanced NSCLC in combination with vinorelbine and cisplatin $(53,54)$. Emerging evidence suggests that endostar in combination with chemotherapy (vinorelbine plus cisplatin) can prolong the time to progression (TTP), and improve the overall response rate (RR) and clinical benefit rate (CBR) with a favorable toxic profile in patients with advanced NSCLC (55). One phase III clinical trial was carried out between April 2003 and July 2004 to investigate the therapeutic effects of endostar in combination with chemotherapy (vinorelbine plus cisplatin) on advanced NSCLC; a total of 486 patients were recruited for the study from 24 medical centers across the country (55). The results demonstrated that the RRs of the experimental group and the control group (NP) were 35.4 and $19.5 \%(\mathrm{P}<0.001)$, the CBRs were 73.3 and $64.0 \%(\mathrm{P}<0.05)$, and the median TTPs were 6.3 and 3.6 months $(\mathrm{P}<0.001)$, respectively. For patients with relapsed NSCLC, the RRs of the endostar in combination with chemotherapy group and the control group were 23.9 and 8.5\% $(\mathrm{P}<0.01)$, the CBRs were 65.2 and $61.7 \%(\mathrm{P}=0.68)$, and the median TTPs were 5.7 and 3.2 months $(\mathrm{P}<0.001)$, respectively. No significant difference was identified in the incidence of moderate to severe adverse reactions in patients between the endostar in combination with chemotherapy group and the control group (55).

Endostar has been used via intracavity injection for the treatment of patients with malignant serous effusions, including MPE and malignant peritoneal effusion. Evidence indicates that endostar alone or in combination with chemotherapy drugs is safe and effective for the treatment of malignant serous effusions in patients with cancer (56-69). Only a few of these clinical studies will be discussed in the present study and others are summarized in Table II. Results from a recent randomized controlled clinical study (56), with 


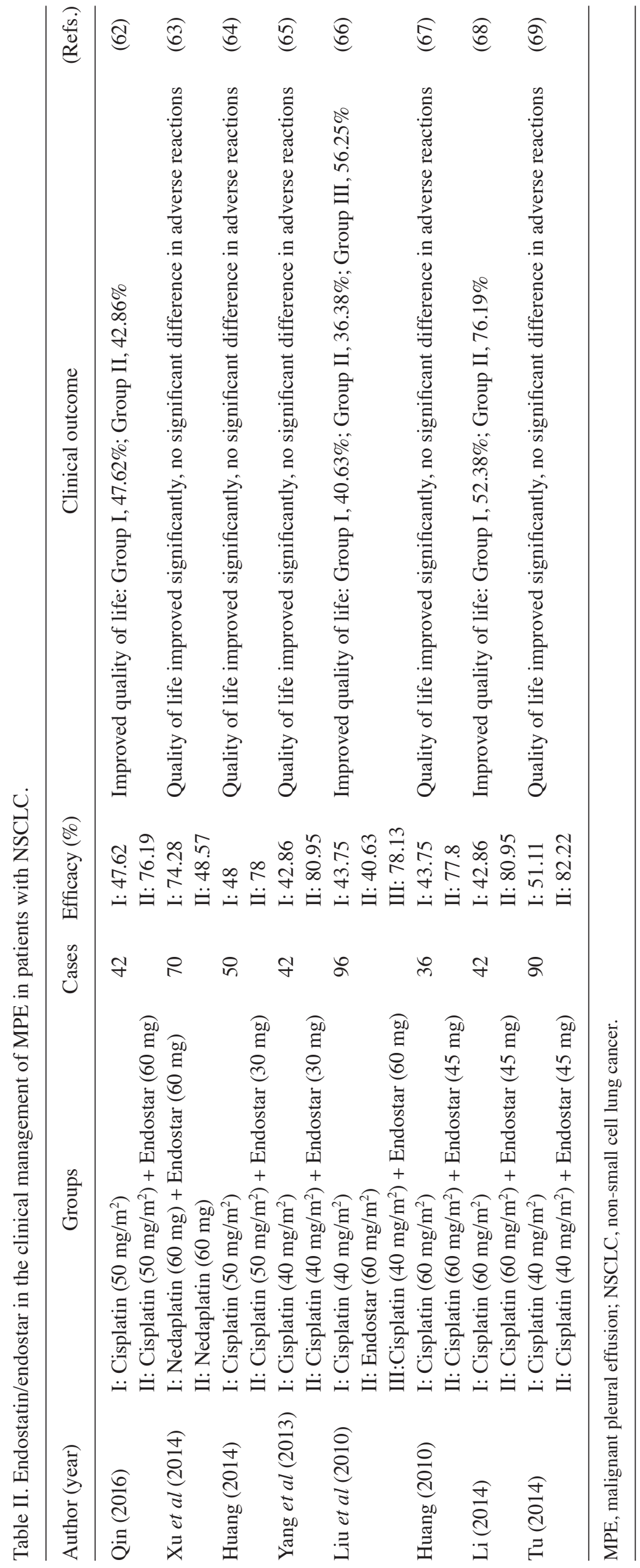




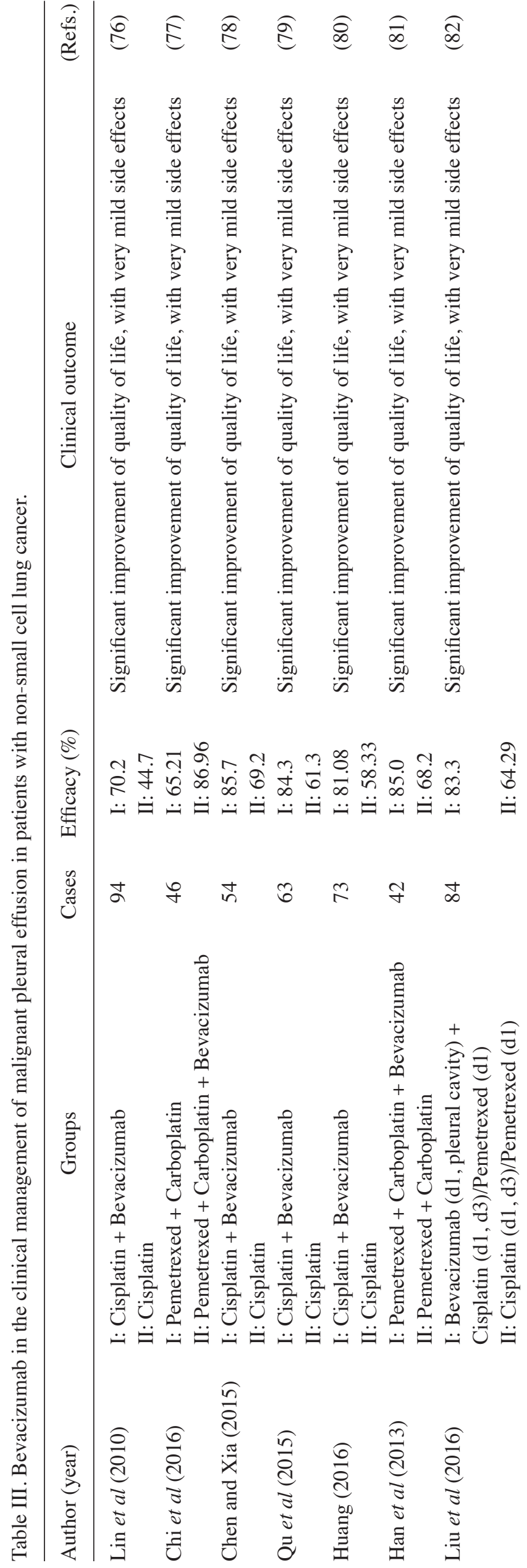

participation from numerous institutions, provided strong evidence that endostar is effective for the treatment of MPE. In addition, Qin et al (56) compared the efficacy of endostar on MPE between patients treated with endostar or cisplatin alone, or with the combination of the two drugs. The analysis revealed that either endostar alone or in combination with cisplatin is effective and safe for the treatment of patients with malignant cavity effusion, with objective response rates (ORRs) of 51,49 and 36\%, for the combination-treated group, endostar-treated group and cisplatin group, respectively. Specifically, for patients with MPE, the ORRs were 62, 58 and $38 \%$ for the combination-treated group, endostar-treated group and cisplatin group, respectively. For patients with ascites, the ORRs were 39, 42 and $32 \%$, respectively (56). These effects of endostar on MPE are supported by results of a similar clinical report by Hu et al (57). Similarly, 63.6\% of patients with MPE demonstrated improvement following treatment with endostar in combination with cisplatin, which was significantly better than cisplatin treatment alone (40.6\%; $\mathrm{P}=0.022$ ). The average progression-free survival (PFS) time for patients with MPE who received treatment with endostar in combination with cisplatin was 95 days, which was significantly longer than that of patients treated with cisplatin alone (PFS, 53 days; $\mathrm{P}=0.039$ ) (57). Endostar has also been demonstrated to inhibit ascites formation and prolong survival in mouse models of malignant ascites established using S180 and $\mathrm{H} 22$ tumor cells (58). The tumor cells collected from the ascites in endostar-treated mice demonstrated a decrease in the expression of VEGF mRNA (58). In addition, treatment of S180 and H22 tumor cells with endostar revealed a significant inhibition of VEGF protein secretion and VEGF mRNA expression, but no effect on cellular proliferation (58). The inhibitory effects of recombinant human endostatin/endostar on tumor growth have also been reported in other cancer types, including ovarian cancer, malignant melanoma and colon cancer, and in liver transplantation-associated angiogenesis (59).

Despite the general inhibitory effects of endostatin/endostar on tumor progression, and on MPE in patients with NSCLC, opinions on the best dosage and administration, and the duration of treatment remain controversial. In most stage II-IV clinical studies, endostar was administered at $30-60 \mathrm{mg} / \mathrm{m}^{2}$, intravenously for 3-4 h/day for 1-14 days. Increasing evidence indicates that the antitumor effects of endostar are time- and dose-dependent; prolongation of administration time and a gradual increase in its blood level can improve its antitumor effects (47). When it was administered intraperitoneally (i.p.) in a single bolus dose to tumor-bearing mice, endostatin was rapidly cleared in the tumor tissues within $2 \mathrm{~h}$, whereas endostatin administered continuously via implanted mini-osmotic pump maintained systemic concentrations of $200-300 \mathrm{ng} / \mathrm{ml}$ for the duration of administration (42). In addition, continuous i.p. administration of endostatin resulted in more effective tumor suppression at significantly reduced doses (5-fold) compared with bolus administration (42). Results of clinical studies have also demonstrated that the antitumor effects of continuous i.p. administration of endostatin/endostar are better compared with the same dose in short-term intravenous administration $(60,61)$. Continuous administration via 
implanted osmotic pump may be able to maintain a stable plasma concentration of endostatin/endostar, so that it can persistently act on newly-formed vascular endothelial cells, resulting in a sustained and constant treatment effect (60). Therefore, continuous administration via an implanted mini-osmotic pump provides a novel method to further improve the therapeutic effects of endostatin/endostar on MPE in patients with NSCLC.

Bevacizumab to target VEGFA. The identification and isolation of VEGFA in 1989 provided a novel avenue for the development of antiangiogenic strategies (9). Consequently, a recombinant humanized monoclonal anti-VEGFA antibody, termed bevacizumab, was generated to block angiogenesis by inhibiting VEGFA. In 2006, the USA Food and Drug Administration approved bevacizumab for use in first-line treatment for advanced nonsquamous NSCLC in combination with carboplatin/paclitaxel chemotherapy. Results of the BEYOND study led by Qingcun Zhou at Tongji University suggested that bevacizumab is safe and effective for the treatment of patients with advanced or recurrent nonsquamous NSCLC in China, including patients with the epidermal growth factor receptor mutation (70). Bevacizumab was then approved by the Chinese Food and Drug Administration for NSCLC in China on July 1, 2015, providing an additional choice for the treatment of MPE in patients with NSCLC.

The antiangiogenic mechanism of bevacizumab. Acting by promoting the formation of new blood vessels and increasing vascular permeability, VEGFA is one of the most important key mediators for the development of MPE, including in patients with NSCLC (12). VEGFA has been demonstrated to induce inflammatory responses and disrupt cell-cell connections to increase vascular permeability and, consequently, promote tumor cell migration. VEGFR1 and VEGFR2, two receptor tyrosine kinases, are the receptors for VEGFA on endothelial cells (71). VEGFR2 may be more important than VEGFR1 for VEGFA-mediated endothelial cell proliferation, angiogenesis and vascular permeability (71). Upon ligation by VEGFA, VEGFR2 can be activated through receptor dimerization and autophosphorylation, thus resulting in activation of various downstream signal cascades (71). Bevacizumab can block the binding of VEGFA to its receptors and thus inhibit activation of the downstream signaling pathways $(72,73)$.

Bevacizumab in the clinical management of MPE in patients with NSCLC. Preclinical evidence suggests that bevacizumab can reduce vascular permeability and decrease the formation of pleural effusion (12). The efficacy of bevacizumab in combination with paclitaxel/carboplatin in the treatment of advanced nonsquamous NSCLC with MPE without chemotherapy was studied in Japan, in a multi-center, clinical phase II prospective study (74). After 2-6 cycles of treatment with bevacizumab in combination with paclitaxel/carboplatin, it was demonstrated that patients exhibited an ORR of $60.8 \%$ and a disease control rate of $87.0 \%$; the disease control rate of MPE was higher compared with in patients who received paclitaxel/carboplatin chemotherapy alone (with a disease control rate of $78.3 \%$ ) (74). Data from two retrospective studies also confirmed that bevacizumab combined with chemotherapy drugs through intravenous injection can effectively control MPE in patients with nonsquamous NSCLC, with a MPE control rate of $92.3 \%$ and a MPE release rate of $\leq 71.4 \%$ (4). The efficacy of bevacizumab combined with platinum through local pleural administration in the treatment of MPE was investigated by Hsu et al (75). The results indicated that local pleural administration of bevacizumab plus cisplatin, alongside the systemic administration of paclitaxel, resulted in a much higher ORR (83.3 vs. 50.0\%; $\mathrm{P}<0.05)$, compared with that in patients who received systemic administration of paclitaxel plus local cisplatin only (75). Patients in the first group demonstrated a significant reduction in the amount of pleural effusion, accompanied with a markedly improved quality of life, and tolerated the treatment well (75). Substantial additional clinical studies have all reported that bevacizumab is safe and effective for the treatment of MPE in patients with NSCLC (Table III) (76-82). Therefore, local administration of bevacizumab in the pleural cavity plus systemic administration of chemotherapy drugs may effectively control MPE in patients with advanced nonsquamous NSCLC.

\section{VEGF in the prognosis of MPE in various subtypes of NSCLC and in patients with advanced NSCLC}

VEGF can promote the occurrence and development of MPE in patients with NSCLC directly (via increasing vascular permeability) and indirectly (via promoting angiogenesis and tumor migration). Accordingly, the therapeutic efficiency of VEGF-targeted strategies for the management of MPE in patients with NSCLC depends on their inhibitory effects on vascular permeability and tumor angiogenesis. The most common types of NSCLC are squamous cell carcinoma, large cell carcinoma and adenocarcinoma, but there are several other types that occur less frequently. All types can occur in unusual histological variants and as mixed cell-type combinations and thus vary in metastatic features and VEGF-VEGFR functional signatures (47). Therefore, various types of NSCLC may differ in their response to VEGF-targeted strategies for the management of associated MPE. Nevertheless, although detailed information is limited in the current literature and more comprehensive clinical studies are required, it appears that endostar and bevacizumab are effective for the treatment of MPE in most NSCLC types, including squamous cell carcinoma and adenocarcinoma (83-85).

It is increasingly clear that levels of VEGF in pleural effusion may be one of the critical indicators of the prognosis of MPE in patients with advanced NSCLC, and anti-VEGF therapy is of important therapeutic value (47). Firstly, VEGF is overexpressed in the majority of patients with advanced NSCLC and MPE, and VEGF levels in pleural effusion are increased and associated with the prognosis of patients with advanced NSCLC and MPE (17-20). A higher concentration of VEGF in pleural effusion implies a higher risk of distant metastasis for patients with NSCLC (75). Specifically, it has been demonstrated that the levels of VEGF and endostatin in pleural effusion, together with the serum levels of endostatin, are prognostic parameters for patients with advanced NSCLC and MPE (17-20,86,87). Secondly, anti-VEGF therapy is safe and effective for patients 
with advanced NSCLC (47). In a number of phase II trials in patients with advanced metastatic NSCLC, the addition of bevacizumab to standard carboplatin/paclitaxel chemotherapy significantly increased the TTP and increased the RR when compared with chemotherapy alone. This was particularly impressive in the subset of patients with non-squamous histology. Bevacizumab is generally well tolerated and does not appear to increase the incidence or severity of nausea/vomiting, neuropathy and renal toxicity, which are typically associated with carboplatin/paclitaxel chemotherapy (74,88-91). Nevertheless, although bevacizumab improves outcomes when added to platinum-based chemotherapy in advanced-stage non-squamous NSCLC, a recent phase III trial study demonstrated that the addition of bevacizumab to adjuvant chemotherapy did not improve overall survival for patients with surgically resected early-stage NSCLC (92). In the future, bevacizumab may be used alongside novel molecular therapies or immuno-oncology drugs, in order to optimize RRs and overcome resistance in patients with advanced NSCLC (93).

\section{Conclusion and perspectives}

VEGF is of great significance to the diagnosis and clinical treatment of MPE in patients with NSCLC. With the recent development of molecular biological technology, great advances have been made in the diagnosis of MPE, including biochemical analysis, cytopathology and imaging examination of pleural effusion. Notably, the pathogenesis of MPE involves numerous factors and complex molecular mechanisms. With an improved understanding of the role for VEGF in the development of MPE, particularly in patients with NSCLC, targeting VEGF has provided a novel strategy for the diagnosis and treatment of patients with MPE. Since approval of the clinical use of endostar and bevacizumab, substantial clinical studies have been conducted worldwide. Results from these studies have provided strong evidence to suggest that endostar and bevacizumab are safe and effective for the treatment of MPE, particularly in patients with NSCLC. With treatment, patients with NSCLC and MPE not only exhibited an improved quality of life but also, to a certain extent, an improved survival rate. It has been speculated that additional clinical studies, particularly well-controlled ones with a larger number of patient cases currently ongoing, may provide additional comprehensive insights for the enhanced judgment of the efficacy of targeting VEGF in patients with NSCLC and MPE.

\section{Acknowledgements}

The authors would like to thank Dr. Xian-Ming Chen (Creighton University, Omaha, NE, USA) for helpful and stimulating discussions.

\section{Funding}

No funding was received.

\section{Availability of data and materials}

All data generated or analyzed during this study are included in this published article.

\section{Authors' contributions}

YC, NWM and HL wrote the paper. All authors read and approved the final manuscript.

\section{Ethics approval and consent to participate}

Not applicable.

\section{Consent for publication}

Not applicable.

\section{Competing interests}

The authors declare that they have no competing interests.

\section{References}

1. Wong MCS, Lao XQ, Ho KF, Goggins WB and Tse SLA: Incidence and mortality of lung cancer: Global trends and association with socioeconomic status. Sci Rep 7: 14300, 2017.

2. Chen W, Zheng R, Baade PD, Zhang S, Zeng H, Bray F, Jemal A, Yu XQ and He J: Cancer Statistics in China, 2015. CA Cancer J Clin 66: 115-132, 2016.

3. Penz E, Watt KN, Hergott CA, Rahman NM and Psallidas I: Management of malignant pleural effusion: Challenges and solutions. Cancer Manag Res 9: 229-241, 2017.

4. Shi H: Guidelines to the diagnosis and treatment of malignant pleural effusion. Chin J Intern Med 53: 166-167, 2014.

5. Zachary I: Signaling mechanisms mediating vascular protective actions of vascular endothelial growth factor. Am J Physiol Cell Physiol 280: C1375-C1386, 2001.

6. Ide AG, Baker NH and Warren SL: Vascularization of the brown pearce rabbit epithelioma transplant as seen in the transparent ear chamber. Am J Roentgenol 42: 891-899, 1939.

7. Folkman J: Tumor angiogenesis: Therapeutic implications. N Engl J Med 285: 1182-1186, 1971.

8. Senger DR, Galli SJ, Dvorak AM, Perruzzi CA, Harvey VS and Dvorak HF: Tumor cells secrete a vascular permeability factor that promotes accumulation of ascites fluid. Science 219: 983-985, 1983.

9. Ferrara $\mathrm{N}$ and Henzel WJ: Pituitary follicular cells secrete a novel heparin-binding growth factor specific for vascular endothelial cells. Biochem Biophys Res Commun 161: 851-858, 1989.

10. de Vries C, Escobedo JA, Ueno H, Houck K, Ferrara N and Williams LT: The fms-like tyrosine kinase, a receptor for vascular endothelial growth factor. Science 255: 989-991, 1992.

11. Harmey JH and Bouchier-Hayes D: Vascular endothelial growth factor (VEGF), a survival factor for tumor cells: Implications for anti-angiogenic therapy. Bioessays 24: 280-283, 2002.

12. Bradshaw M, Mansfield A and Peikert T: The role of vascular endothelial growth factor in the pathogenesis, diagnosis and treatment of malignant pleural effusion. Curr Oncol Rep 15: 207-216, 2013.

13. Zang J, Hu Y, Xu X, Ni J, Yan D, Liu S, He J, Xue J, Wu J and Feng J: Elevated serum levels of vascular endothelial growth factor predict a poor prognosis of platinum-based chemotherapy in non-small cell lung cancer. Onco Targets Ther 10: 409-415, 2017.

14. Popper HH: Progression and metastasis of lung cancer. Cancer Metastasis Rev 35: 75-91, 2016.

15. Hu CP: Interpretation of expert consensus 2014 on diagnosis and treatment of malignant pleural effusion. J Transl Int Med 3: 1-2, 2015.

16. Dvorak HF: Vascular permeability factor/vascular endothelial growth factor: A critical cytokine in tumor angiogenesis and a potential target for diagnosis and therapy. J Clin Oncol 20: 4368-4380, 2002.

17. Fafliora E, Hatzoglou C, Gourgoulianis KI and Zarogiannis SG: Systematic review and meta-analysis of vascular endothelial growth factor as a biomarker for malignant pleural effusions. Physiol Rep 4: e12978, 2016. 
18. Sriram KB, Relan V, Clarke BE, Duhig EE, Yang IA, Bowman RV Lee YC and Fong KM: Diagnostic molecular biomarkers for malignant pleural effusions. Future Oncol 7: 737-752, 2011.

19. Gu Y, Zhang M, Li GH, Gao JZ, Guo L, Qiao XJ, Wang LH, He L, Wang ML, Yan L and Fu XH: Diagnostic values of vascular endothelial growth factor and epidermal growth factor receptor for benign and malignant hydrothorax. Chin Med J (Engl) 128: 305-309, 2015

20. Zhou WB, Bai M and Jin Y: Diagnostic value of vascular endothelial growth factor and endostatin in malignant pleural effusions. Int J Tunerc Lung Dis 13: 381-386, 2009.

21. Nam HS: Malignant pleural effusion: Medical approaches for diagnosis and management. Tuberc Respir Dis (Seoul) 76 : 211-217, 2014.

22. Chen Y, Liang B, Zhao YJ, Wang SC, Fan YB and Wu GP: Transcription expression and clinical significance of vascular endothelial growth factor mRNA and endostatin mRNA in pleura effusions of patients with lung cancer. Diagn Cytopathol 40: 287-291, 2012

23. Jiang B, Wu GP, Zhao YJ and Wang SC: Transcription expression and clinical significance of TTF-1 mRNA in pleural effusion of patients with lung cancer. Diagn Cytopathol 36: 849-854, 2008.

24. Palaoro LA, Blanco AM, Gamboni M, Rocher AE and Rotenberg RG: Usefulness of ploidy, AgNor and immunocytochemistry for differentiating benign and malignant cells in serous effusions. Cytopathology 18: 33-39, 2007.

25. Light RW: Pleural effusions. Med Clin North Am 95: 1055-1070, 2011.

26. Liu QF: Clinical value of VEGF combined with CEA in the treatment of malignant pleural effusion of advanced NSCLC. Practical Oncol J (China) 27: 156-159, 2013.

27. Stathopoulos GT and Kalomenidis I: Malignant pleural effusion: Tumor-host interactions unleashed. Am J Respir Crit Care Med 186: 487-492, 2012

28. Hamed EA, El-Noweihi AM, Mohamed AZ and Mahmoud A: Vasoactive mediators (VEGF and TNF-alpha) in patients with malignant and tuberculous pleural effusions. Respirology 9 : 81-86, 2004.

29. Gerber HP and Ferrara N: Pharmacology and pharmacodynamics of bevacizumab as monotherapy or in combination with cytotoxic therapy in preclinical studies. Cancer Res 65: 671-680, 2005.

30. Numnum TM, Rocconi RP, Whitworth J and Barnes MN: The use of bevacizumab to palliate symptomatic ascites in patients with refractory ovarian carcinoma. Gynecol Oncol 102: 425-428, 2006

31. Ferrara N, Gerber HP and LeCouter J: The biology of VEGF and its receptors. Nat Med 9: 669-676, 2003.

32. Hicklin DJ and Ellis LM: Role of the vascular endothelia growth factor pathway in tumor growth and angiogenesis. J Clin Oncol 23: 1011-1027, 2005.

33. Pritchard-Jones RO, Dunn DB, Qiu Y, Varey AH, Orlando A, Rigby H, Harper SJ and Bates DO: Expression of VEGF (XXX) $\mathrm{b}$, the inhibitory isoforms of VEGF, in malignant melanoma. Br J Cancer 97: 223-230, 2007.

34. Fontanini G, Lucchi M, Vignati S, Mussi A, Ciardiello F, De Laurentiis M, De Placido S, Basolo F, Angeletti CA and Bevilacqua G: Angiogenesis as a prognostic indicator of survival in nonsmall-cell lung carcinoma: A prospective study. J Natl Cancer Inst 89: 881-886, 1997.

35. Ferrara N: Vascular endothelial growth factor: Basic science and clinical progress. Endocr Rev 25: 581-611, 2004.

36. Voelkel NF, Vandivier RW and Tuder RM: Vascular endothelial growth factor in the lung. Am J Physiol Lung Cell Moistiol 290: L209-L221, 2006.

37. Semenza GL: Targeting HIF-1 for cancer therapy. Nat Rev Cancer 3: 721-732, 2003.

38. Mayerhofer M, Valent P, Sperr WR, Griffin JD and Sillaber C: $\mathrm{BCR} / \mathrm{ABL}$ induces expression of vascular endothelial growth factor and its transcriptional activator, hypoxia induciblefactor-1alpha, through a pathway involving phosphoinositide 3 -kinase and the mammalian target of rapamycin. Blood 100 3767-3775, 2002

39. Loeffler S, Fayard B, Weis J and Weissenberger J: Interleukin-6 induces transcriptional activation of vascular endothelial growth factor (VEGF) in astrocytes in vivo and regulates VEGF promoter activity in glioblastoma cells via direct interaction between STAT3 and Sp1. Int J Cancer 115: 202-213, 2005.

40. Cohen T, Nahari D, Cerem LW, Neufeld G and Levi BZ: Interleukin 6 induces the expression of vascular endothelial growth factor. J Biol Chem 271: 736-741, 1996.
41. Wójcik E, Jakubowicz J, Skotnicki P, Sas-Korzyńska B and Kulpa JK: IL-6 and VEGF in small cell lung cancer patients. Anticancer Res 30: 1773-1778, 2010.

42. Yan J, Jiang Y and Xu H: Endu treatment of malignant pleural effusion. Practical J Cancer 27: 538-539, 2012.

43. O'Reilly MS, Boehm T, Shing Y, Fukai N, Vasios G, Lane WS, Flynn E, Birkhead JR, Olsen BR and Folkman J: Endostatin: An endogenous inhibitor of angiogenesis and tumor growth. Cell 88: 277-285, 1997.

44. Hayes AJ, Li LY and Lippman ME: Science, medicine, and the future. Antivascular therapy: A new approach to cancer treatment. BMJ 318: 853-856, 1999.

45. Xu X, Mao W, Chen Q, Zhuang Q, Wang L, Dai J, Wang H and Huang Z: Endostar, a modified recombinant human endostatin, suppresses angiogenesis through inhibition of $\mathrm{Wnt} / \beta$-catenin signaling pathway. PLoS One 9: e107463, 2014

46. Rong B, Yang S, Li W, Zhang W and Ming Z: Systematic review and meta-analysis of Endostar (rh-endostatin) combined with chemotherapy versus chemotherapy alone for treating advanced non-small cell lung cancer. World J Surg Oncol 10: 170, 2012.

47. Mohajeri A, Sanaei S, Kiafar F, Fattahi A, Khalili M and Zarghami $\mathrm{N}$ : The challenges of recombinant endostatin in clinical application: Focus on the different expression systems and molecular bioengineering. Adv Pharm Bull 7: 21-34, 2017

48. Shichiri M and Hirata Y: Antiangiogenesis signals by endostatin. FASEB J 15: 1044-1053, 2001.

49. Hajitou A, Grignet C, Devy L, Berndt S, Blacher S, Deroanne CF, Bajou K, Fong T, Chiang Y, Foidart JM and Noël A: The antitumoral effect of endostatin and angiostatin is associated with a down-regulation of vascular endothelial growth factor expression in tumor cells. FASEB J 16: 1802-1804, 2002.

50. Li DH, Liu QG, Wang HP, Yu M, Jiang J, Tang CH and Ning YL: Study on recombinant human endostatin in the treatment of malignant pleural effusion in the elderly. Shaanxi Med J 44: 1138-1139, 2015 (In Chinese).

51. Murakami A, Tabata C, Tabata R, Okuwa $\mathrm{H}$ and Nakano $\mathrm{T}$ : Clinical role of pleural effusion MMP-3 levels in malignant pleural mesothelioma. Oncol Lett 3: 581-585, 2012.

52. Ma X, Yao Y, Yuan D, Liu H, Wang S, Zhou C and Song Y: Endostar suppresses angiogenesis and lymphangiogenesis of malignant pleural effusion in mice. PLoS One 7: e53449, 2012.

53. Ishii H, Yazawa T, Sato H, Suzuki T, Ikeda M, Hayashi Y, Takanashi Y and Kitamura H: Enhancement of pleural treatment and lymph node metastasis of intrathoracic lung cancer cells by vascular endothelial growth factors (VEGFs). Lung Cancer 45: 325-337, 2004

54. Prager GW, Lackner EM, Krauth MT, Unseld M, Poettler M, Laffer S, Cerny-Reiterer S, Lamm W, Kornek GV, et al: Targeting of VEGF dependent transendothelial migration of cancer cells by bevacizumab. Mol Oncol 4: 150-160, 2010.

55. Wang J, Sun Y, Liu Y, Yu Q, Zhang Y, Li K, Zhu Y, Zhou Q, Hou M, Guan Z, et al: Results of randomized, multicenter, double-blind phase III trial of rh-endostatin (YH-16) in treatment of advanced non-small cell lung cancer patients. Zhongguo Fei Ai Za Zhi 8: 283-290, 2005 (In Chinese).

56. Qin S, Yang L, Liang J, Cheng Y, Tan Q, Bi J, Wang L, Hu B, Shi J, Sun G, et al: Prospective, randomized, multicenter clinical study of endometrial and cisplatin in the treatment of malignant pleural effusion. Chin J Clin Oncol 22: 193-202, 2017 (In Chinese).

57. Hu X, Shi Y, Wang H, Zhang C, Liu P, Wang Y and Li J: A clinical study on intra-thoracic chemotherapy of recombinant human endostatin combined with cisplatin for malignant pleural effusion. Clin Med J 3: 23-27, 2015.

58. Wei H, Qin S, Yin X, Chen Y and Hua H: Endostar inhibits ascites formation and prolonged survival in mouse models of malignant ascites. Oncol Lett 9: 2694-2700, 2015.

59. Qin S, Liu X, Wang L, Chen Y, Qian J, Hui H, Gong X, Yang L and He Z: Clinical study of recombinant human endostatin combined with chemotherapy in the treatment of advanced malignant tumors of lung. Clin J Onol 34: 426-428, 2012 (In Chinese).

60. Kisker O, Becker CM, Prox D, Fannon M, D'Amato R, Flynn E, Fogler WE, Sim BK, Allred EN, Pirie-Shepherd SR and Folkman J: Continuous administration of endostatin by intraperitoneally implanted osmotic pump improves the efficacy and potency of therapy in a mouse xenograft tumor model. Cancer Res 61: 7669-7674, 2011. 
61. Shen H, Zhao J, Weng SS, Fang XF, Zhang YY and Huang JJ: Continuous administration of endostar plus GP chemotherapy in local advanced or metastatis lung squamous cell carcinoma. Acta Medica Mediterr 32: 57-62, 2016.

62. Qin M: Clinical observation of cisplatin plus endostar endovascular infusion in the treatment of advanced non-small cell lung cancer with malignant pleural effusion. China Pract Med 11: 228-229, 2016

63. Xu J, Qi DL, Li XB and Wang RX: Recombinant human endostatin combined with chemotherapy in the treatment of malignant pleural effusion of non-small cell lung cancer. Chin J Clin Oncol 24: 1573-1576, 2014.

64. Huang L: Clinical observation on treatment of malignant pleural effusion of Non-small cell lung cancer with. Jilin Med 35: 4308-4309, 2014.

65. Yang Y, Lin R and Cao G: Combined with cisplatin in the treatment of malignant pleural effusion in non-small cell lung cancer. Chin Med 22: 21-22, 2013.

66. Liu W, Ham M, Yin N and Li J: Clinical study on the treatment of non-small cell lung cancer with malignant pleural effusion by intrapleural injection with. Shandong Pharmaceutical 50: 79-80, 2010.

67. Huang J: Observation on the therapeutic effect of Endu combined with cisplatin intrapleural injection on malignant pleural effusion. J Clin Med Practical 13: 63-64, 2010.

68. Li W: Observation of curative effect of intrapleural perfusion of malignant pleural effusion. J Med Forum 32: 170-172, 2011.

69. Tu J: Clinical study of recombinant human endostatin combined with intrapleural injection of cisplatin in the treatment of malignant pleural effusion of non-small cell lung cancer. Pract J Cancer 12: 1592-1594, 2014 (In Chinese)

70. Zhou C, Wu YL, Chen G, Liu X, Zhu Y, Lu S, Feng J, He J, Han B, Wang J, et al: BEYOND: A randomized, double-blind, placebo-controlled, multicenter, phase III study of first-line carboplatin/paclitaxel plus bevacizumab or placebo in Chinese patients with advanced or recurrent nonsquamous non-small-cell lung cancer. J Clin Oncol 33: 2197-2204, 2015.

71. Shibuya M: Vascular endothelial growth factor (VEGF) and its receptor (VEGFR) signaling in angiogenesis: A crucial target for anti- and pro-angiogenic therapies. Genes Cancer 2: 1097-1105, 2011.

72. Wu Z, Wu Q, Sun D, Wang Z, Shi Y and Dai G: Progress in response prediction for Bevacizumab in anti-tumor therapy. Prog Mod Bio 14: 4570-4573, 2014.

73. Guan F, Li Z, Yuan S and Gao J: Current status and clinical application of bevacizumab. Chin Sci 12: 30, 2016.

74. Tamiya M, Tamiya A, Yamadori T, Nakao K, Asami K, Yasue T, Otsuka T, Shiroyama T, Morishita N, Suzuki H, et al: Phase 2 study of bevacizumab with carboplatin-paclitaxel for non-small cell lung cancer with malignant pleural effusion. Med Oncol 30: 676, 2013.

75. Hsu LH, Hsu PC, Liao TL, Feng AC, Chu NM and Kao SH: Pleural fluid osteopontin, vascular endothelial growth factor and urokinase-type plasminogen activator levels as predictors of pleurodesis outcome and prognosticators in patients with malignant pleural effusion: A prospective cohort study. BMC Cancer 16: 463, 2016.

76. Lin FH, Su WP and Jin R: Clinical study of bevacizumab combined with cisplatin in the treatment of malignant pleura effusion of non-small cell lung cancer. Clin J Clin Med 44: 698-700, 2010 (In Chinese).

77. Chi J, Bai Y and Chen H: Clinical study of bevacizumab combined with carboplatin in the treatment of malignant pleural effusion of non-small cell lung cancer. Chin J Clin Pharmacol 13: 1175-1180, 2016 (In Chinese).

78. Chen L and Xia SY: Effects and safety of bevacizumab combined with cisplatin in the treatment of malignant pleural effusion of non-small cell lung cancer. J Shanghai Jiaotong Univ 8 : $1194-1198,2015$

79. Qu B, Jiang W and Zhou Z: Clinical study of bevacizumab combined with cisplatin in the treatment of malignant pleural effusion of non-small cell lung cancer. J Chin Med Univ 7: 648-652, 2015 (In Chinese).
80. Huang B: Efficacy of bevacizumab combined with cisplatin in the treatment of malignant pleural effusion in non-small cell lung cancer. Intern J Resp Dis 11: 814-817, 2016.

81. Han N, Zhang MX, Yu SY and Cheng Y: Clinical study of bevacizumab combined with cisplatin/pemetrexed in the treatment of malignant pleural effusion of non-squamous cell carcinoma of non-squamous cell carcinoma. J Huazhong Univ Sci Tech 5: 588-591, 2013.

82. Liu HP, Gong CP, Qu L, Li X, Hu HT and Zhang YL: Efficacy of bevacizumab combined with cisplatin/pemetrexed in the treatment of malignant pleural effusion of non-small cell lung cancer. Med J Nat Defending Forces Southwest Chin 12: 1148-1151, 2016.

83. Lu J, Xie Q, Chen Q, Sun WH, Zhong AH, Shi Q, Liao SX and Zhu JX: The expression of BRMS1 in lung cancer and its effect on invasion and metastasis of lung cancer cells. J Clin Pulmonary Med 9: 1664-1667, 2016.

84. Usui K, Sugawara S, Nishitsuji M, Fujita Y, Inoue A, Mouri A, Watanabe H, Sakai H, Kinoshita I, Ohhara Y, et al: A phase II study of bevacizumab with carboplatin-pemetrexed in non-squamous non-small cell lung carcinoma patients with malignant pleural effusions: North East Japan Study Group Trial NEJ013A. Lung Cancer 99: 131-136, 2016.

85. Masago K, Fujimoto D, Fujita S, Hata A, Kaji R, Ohtsuka K, Okuda C, Takeshita J and Katakami N: Response to bevacizumab combination chemotherapy of malignant pleural effusions associated with non-squamous non-small-cell lung cancer. Mol Clin Oncol 3: 415-419, 2015

86. Tamiya M, Tamiya A, Yasue T, Nakao K, Omachi N, Shiroyama T, Tani E, Hamaguchi M, Morishita N, Suzuki H, et al: Vascular endothelial growth factor in plasma and pleural effusion is a biomarker for outcome after bevacizumab plus carboplatin-paclitaxel treatment for non-small cell lung cancer with malignant pleural effusion. Anticancer Res 36: 2939-2944, 2016.

87. Gkiozos I,TsagouliS, Charpidou A,GrapsaD,KainisE,GratziouC and Syrigos K: Levels of vascular endothelial growth factor in serum and pleural fluid are independent predictors of survival in advanced non-small cell lung cancer: Results of a prospective study. Anticancer Res 35: 1129-1137, 2015.

88. Zhang Y, Yu LK, Lu GJ, Xia N, Xie HY, Hu W, Hao KK, Xu CH and Qian Q: Prognostic values of VEGF and Endostatin with malignant pleural effusions in patients with lung cancer. Asian Pac J Caner Prev 15: 8435-8440, 2014

89. Hooper CE, Elvers KT, Welsh GI, Millar AB and Maskell NA: VEGF and sVEGFR-1 in malignant pleural effusions: Association with survival and pleurodesis outcomes. Lung Cancer 77: 443-449, 2012

90. Sandler AB, Johnson DH and Herbst RS: Anti-vascular endothelial growth factor monoclonals in non-small cell lung cancer. Clin Cancer Res 10: 4258-4262, 2004

91. Kitamura K, Kubota K, Ando M, Takahashi S, Nishijima N, Sugano T, Toyokawa M, Miwa K, Kosaihira S, Noro R, et al: Bevacizumab plus chemotherapy for advanced non-squamous non-small-cell lung cancer with malignant pleural effusion. Cancer Chemother Pharmacol 71: 457-461, 2013

92. Wakelee HA, Dahlberg SE, Keller SM, Tester WJ, Gandara DR, Graziano SL, Adjei AA, Leighl NB, Aisner SC, Rothman JM, et al: Adjuvant chemotherapy with or without bevacizumab in patients with resected non-small-cell lung cancer (E1505): An open-label, multicentre, randomised, phase 3 trial. Lancet Oncol 18: 1610-1623, 2017.

93. Assoun S, Brosseau S, Steinmetz C, Gounant V and Zalcman G: Bevacizumab in advanced lung cancer: State of the art. Future Oncol 13: 2515-2535, 2017.

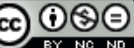

This work is licensed under a Creative Commons Attribution-NonCommercial-NoDerivatives 4.0 International (CC BY-NC-ND 4.0) License. 